\title{
How valuable are urinary prostate-specific antigen levels in detecting recurrence of disease?
}

\author{
Robert Nam, MD, FRCSC
}

See related article on page 213

$\mathrm{T}$ he study by Bolduc and colleagues ${ }^{1}$ generates an interesting hypothesis as to whether the measurement of urinary prostate-specific antigen (UPSA) levels after treatment with surgery can identify patients who experience local recurrence earlier than conventional serum PSA measurements. The clinical significance of this question could have an important impact on the debate between the use of adjuvant or salvage radiotherapy after radical prostatectomy.

Although many interesting trends were observed in the study, no firm conclusions can be made, as the study sample size was 50 patients. Although follow-up was long, the distribution of grade and stage suggests a highly selected population. Further research is needed to examine whether uPSA levels are correlated to margin status. The examination of the Mantel-Haenzel odds ratio in predicting recurrence based on uPSA levels was high, but given that 2 of the cells were zero, adjustment of the odds ratio may be problematic. An interesting finding of the paper is the Kaplan-Meier estimates of recurrence rates. As the authors observe, the curves are uniform for the first 4 years, but then they diverge. Patients who had positive uPSA levels after surgery appeared to experience a high rate of recurrence compared with patients who had negative uPSA lev- els beyond 4 years. This finding could still be due to chance, small sample size or short follow-up, since it did not reach statistical significance. However, these results are consistent with the biological rationale of measuring uPSA levels and the potential prognostic abilities.

The authors appropriately mention the need for rigorous prospective trials to better examine this hypothesis. If confirmed, measuring uPSA levels could provide important prognostic information and could be instrumental in the role of adjuvant or salvage radiotherapy or even systemic therapy in the management of clinically localized prostate cancer.

Associate Professor of Surgery, Division of Urology, Sunnybrook Health Sciences Centre, University of Toronto, Toronto, Ont.

Competing interests: None declared.

Reference

1. Bolduc $S$, Inman BA, Lacombe L, et al. Early detection of prostate cancer local recurrence by urinary prostate-specific antigen Can Urol Assoc J 2009;3:213-7.

Correspondence: Dr. Robert Nam, Sunnybrook Health Sciences Centre, University of Toronto, 2075 Bayview Ave., MG-406, Toronto ON M4N 3M5; robert.nam@utoronto.ca 